\title{
Medicina e feminização em universidades brasileiras: 0 gênero nas interseções
}

Resumo: Este artigo analisa o perfil socioeconômico, geracional e étnico dos/as estudantes e aspirantes dos cursos de graduação em Medicina em dois estados brasileiros: Bahia e Santa Catarina, entre 2005 e 2015. A abordagem interseccional respalda-se nas contribuições de autoras do campo de Gênero e Ciências, bem como nos estudos sobre a participação de mulheres na área da medicina no Brasil, de modo a contribuir para o debate sobre a feminização da área. Considerando as interferências regionais na configuração dos perfis encontrados em universidades públicas (UFBA e UFSC), bem como em universidades da rede privada (FTC e EBMSP, na Bahia, e UNIVALI e UNESC, em Santa Catarina), a metodologia da pesquisa incluiu o levantamento de dados secundários (sobre sexo, renda familiar, idade, cor e origem), a análise de documentos e a revisão da literatura.

Palavras-chave: gênero; interseções; feminização; medicina

\section{Introdução}

Nos países ocidentais tem sido crescente a participação das mulheres nas carreiras acadêmicas, inclusive na área da Medicina, conforme apontam os estudos de Nathalie LAPEYRE e Nicky Le FEUVRE (2005), na França; Maria do Céu Soares MACHADO (2003), em Portugal; Elianne RISKA (2001) e Magdalena ROSENDE (2008), sobre distintos países europeus, entre outros. Pesquisas realizadas em trinta países pela Organização para Cooperação e Desenvolvimento Econômico (OCDE, 2009) revelaram que as mulheres são maioria desde os anos 2000 nas escolas de medicina dos Estados Unidos e do Canadá, do mesmo modo que constataram um processo de feminização acelerada em países europeus, tais como Inglaterra, Irlanda e Noruega.

Analisando a situação no contexto brasileiro, entre os anos de 1910 e 2010, Mário SCHEFFER e Alex Jones F. CASSENOTE (2013) consultaram e sistematizaram os dados dos

\section{(c) (7)}

Esta obra está sob licença Creative Commons. 
vinte e sete Conselhos Regionais de Medicina, da Comissão Nacional de Residência Médica e da Associação Médica Brasileira e concluíram que a presença das mulheres sofreu um incremento gradativo nesse período, destacando que "[...] desde 2009, entre os novos médicos em atividade, os homens ainda predominam $(60,1 \%)$, mas no grupo com 29 anos ou menos as mulheres já são maioria" (p. 168).

O ingresso das mulheres no ensino superior brasileiro teve início no final do século XIX, expandindo-se de modo significativo em várias carreiras a partir dos anos setenta do século XX, gerando um processo que tem sido chamado de feminização. Com esse termo, entendemos que a literatura especializada se refere não apenas a um aumento estatístico, mas às interferências de gênero nas escolhas e no exercício das atividades profissionais. Consideramos ainda que tal processo é tributário de vários fatores, notavelmente os avanços da escolarização das mulheres, apontados em pesquisas de Nara AZEVEDO e Luiz Otávio FERREIRA (2006), bem como em um estudo posterior liderado por este último em 2008, por exemplo.

A primeira pesquisa constata que as políticas educacionais implantadas no Brasil a partir da década de 1920, gradativamente institucionalizadas no período Vargas, favoreceram a inserção profissional das mulheres na academia e na produção do conhecimento, tendo impacto significativo nos sistemas de gênero (AZEVEDO; FERREIRA, 2006). A segunda dá continuidade ao tema e investiga a crescente escolarização das mulheres no nível superior a partir dos anos 40 , indicando suas repercussões na institucionalização do conhecimento científico e na profissionalização da pesquisa. Os/as autores analisam artigos de quatro revistas científicas publicadas entre os anos de 1939 e 1969, observando que, embora aqueles elaborados pelas mulheres não atingissem nem mesmo a metade da produção masculina, constituíam um avanço em relação aos períodos anteriores (FERREIRA, AZEVEDO, Moema GUEDES, Bianca CORTES, 2008).

Na década anterior, Maria Helena Bueno TRIGO (1994) constatou a importância da criação da Faculdade de Filosofia, Ciências e Letras da Universidade de São Paulo (USP), em 1934, para o ingresso das mulheres na academia e as consequentes mudanças de valores de gênero dentro e fora dessa instituição. $O$ estudo sugere justamente que essa entrada em certas carreiras exerce, direta ou indiretamente, influência sobre escolhas e projetos profissionais, contribuindo para uma visão mais ampla das oportunidades e uma revisão dos valores tradicionais.

Em um livro que se tornou um marco referencial na área, Fanny TABAK (2002) afirmou que até os anos 90 do século XX as carreiras científicas e tecnológicas ainda não eram uma prioridade para as estudantes do segundo grau. No caso da Medicina, a autora ressalta a feminização do campo a partir dos anos 70 , lembrando, por exemplo, que na Universidade Federal do Rio de Janeiro, apenas $35 \%$ do contingente do curso de graduação eram compostos por mulheresno ano de 1977, ao passo que em1990 a proporção quase dobrava, chegando a $62 \%$. Os dados levantados pela autora nessa instituição mostraram que uma situação semelhante ocorreu nas Engenharias, outra área ocupada majoritariamente pelos homens.

Moema de Castro Guedes (2008), em uma perspectiva também histórica, focalizou a presença das mulheres nos cursos universitários e nas pós-graduações, situando-a no contexto das grandes mudanças da sociedade brasileira. A autora analisou os resultados de quatro Censos Demográficos do IBGE, de 1970 a 2000, constatando que, neste período, as mulheres já representavam a maioria $(60 \%)$ dos formados entre os mais jovens. Os dados confirmavam também uma "expressiva entrada feminina em cursos tradicionalmente masculinos" (GUEDES, 2008, p. 117). 
Além dessas contribuições, a elaboração deste trabalho leva em conta também que o aumento expressivo da participação das mulheres na academia e na produção do conhecimento científico é tributário das conquistas dos movimentos feministas, que sem dúvida impulsionaram direta ou indiretamente várias mudanças a nível mundial, favorecendo uma maior igualdade de gênero - juntamente com outros fatores. Conforme aponta o Relatório sobre o Desenvolvimento Mundial (BANCO MUNDIAL, 2011), entre os avanços nesse sentido se destacam: o aumento da expectativa de vida para mulheres, atingindo 71 anos em média global, contra 67 anos para os homens; a queda do número de filhos por mulher, mesmo nos países mais pobres; um expressivo aumento do número de matrículas escolares de meninas e mulheres na educação fundamental, secundária e no ensino superior, a partir de 1970.

Políticas educacionais como transferências condicionais de renda, implantadas em mais de trinta países, também têm contribuído nessa luta pela igualdade, sendo muitas delas explicitamente voltadas para crianças em geral e meninas em particular (BANCO MUNDIAL, 2011 ). Essas e outras políticas, bem como as mudanças na formação das famílias, resultantes do casamento tardio e da fertilidade reduzida, entre outros fatores, tiveram um impacto significativo sobre a participação das mulheres na força de trabalho, registrandose em dez países da América Latina um aumento de aproximadamente dois terços dessa participação. Devido ao aumento da renda que ocorreu em muitos países, desde os anos 80, "a taxa de participação feminina em cada nível de renda tem aumentado acentuadamente ao longo do tempo" (BANCO MUNDIAL, 201 1, p. 12).

Evidentemente, o Relatório também destaca a persistência dos 'hiatos de gênero'l e das desigualdades de gênero² (entre elas a segregação e as disparidades salariais), elaborando assim várias recomendações para uma agenda global para sua superação, na qual as políticas educacionais de estímulo à formação profissional das mulheres devem ocupar um lugar central (BANCO MUNDIAL, 2011).

No Brasil, dados atuais do IBGE (2012) também sinalizam avanços importantes coexistindo com as desigualdades. Uma análise do perfil educacional das mulheres no mercado nacional de trabalho revelou que, em 2009, 61,2\% das trabalhadoras haviam atingido onze anos ou mais de estudos (com pelo menos o ensino médio completo), enquanto que, no caso dos homens, o percentual correspondia a 53,2\%. Assim como a "parcela de mulheres ocupadas com curso de nível superior completo era de $19,6 \%$, superior ao dos homens (14,2\%) [...] nos grupos com anos de estudos com menos escolaridade, a participação dos homens era superior a das mulheres" (IBGE, 2012, p. 05).

Segundo a Pesquisa Mensal de Emprego, as mulheres eram maioria entre estudantes universitários dos 18 aos 24 anos no ano de 2010, correspondendo a $57,1 \%$ do total nessa faixa etária. Considerando a população total nessa faixa, as mulheres também estão em vantagem, com $15,1 \%$ contra $11,4 \%$ de homens. Uma diferença maior foi encontrada no nível superior completo, no qual $12,5 \%$ das mulheres concluíram cursos de graduação, contra $9,9 \%$ dos homens. Coincidindo com o balanço mundial a respeito dos hiatos de gênero, a pesquisa constatou também que, no Brasil, não obstante os avanços, persistem

1 Segundo o Relatório (BANCO MUNDIAL, 2011, p. 12), os hiatos de gênero permanecem grandes para as populações seriamente desfavorecidas, "onde a pobreza é combinada com outros fatores de exclusão, tais como etnia, casta, distância, raça, deficiência física ou orientação sexual".

${ }^{2}$ Entre elas: maior participação das mulheres em atividades de baixa produtividade do mercado informal, com salários mais baixos; alta mortalidade de meninas e mulheres, materna, epidemias (AIDS) etc.; persistência de diferenças de gênero na utilização do tempo, no acesso a bens e créditos e no tratamento dado pelo mercado e instituições formais; menos voz para as mulheres na tomada de decisões nos domicílios e na sociedade (BANCO MUNDIAL, 2011). 
desigualdades de rendimento, principalmente entre as pessoas mais escolarizadas, e os homens continuam recebendo salários mais altos do que as mulheres (IBGE, 2010).

Considerando os resultados desses estudos e visando a contribuir para os debates e para as políticas educacionais, esta pesquisa focaliza a feminização da carreira médica a partir de uma perspectiva de gênero, analisando o perfil socioeconômico, geracional e étnico dos/as estudantes, aspirantes e egressos/as dos cursos de graduação em Medicina das redes pública e privada de ensino, em dois estados brasileiros - Bahia e Santa Catarina. ${ }^{3}$ A investigação nesses estados permitirá identificar algumas semelhanças e diferenças entre diferentes contextos regionais do país.

\section{Gênero e Ciências: mulheres na carreira médica}

A abordagem adotada e detalhada no próximo tópico situa-se no campo que vem sendo denominado mais amplamente de Gênero e Ciências. ${ }^{4}$ Torna-se importante evidenciar que uma revisão precedente da literatura no Brasil (Luzinete Simões MINELLA, 2013 b) procurou identificar as tendências de investigação e observou que a maioria dos estudos tem sido influenciada por algumas obras de referência, produzidas principalmente no contexto anglo-saxão dos anos 80 do último século, nas quais são elaboradas várias críticas feministas às interferências de gênero nas construções científicas. Entre elas, as de Donna HARAWAY (1994; 1995), Sandra HARDING (1996), Londa SCHIEBINGER (2001), Evelyn FOX KELLER e Helen LONGINO (1996) e Fox Keller (2001), autoras que também têm inspirado um debate sistematizado por Maria Margaret LOPES (1998; 2006a; 2006b) - uma das pioneiras da área no Brasil, entre outras.

O presente estudo pode ser identificado mais especificamente com uma das vertentes do campo citado, que inclui investigações sobre a participação das mulheres nas carreiras científicas, o acesso, as escolhas profissionais, a permanência e as interferências de gênero em sua construção. No que se refere aos debates no Brasil, destacamos significativas contribuições de publicações pioneiras da Fundação Carlos Chagas, publicadas na década de 1970; inúmeros artigos publicados pelos Cadernos Pagu, a partir do final dos anos 90; pelos Cadernos de Gênero e Tecnologia, do Centro Federal de Educação Tecnológica (CEFET/Pr) e pela Revista Tecnologia e Sociedade, da Universidade Tecnológica Federal do Paraná (PPGTE/UTFPr). Inspiram ainda vários artigos publicados nessas e em outras fontes, de autoras como Hildete Pereira de MELO (2010), Melo e Maria Carolina Pereira CASEMIRO (2004), Melo e Helena Maria Martins LASTRES (2006), Melo e André Barbosa OLIVEIRA (2006), Melo e Lígia Maria RODRIGUES (2006), Carla Giovana CABRAL e Walter Antonio BAZZO (2005), CABRAL (2006a; 2006b; 2008), Maria Rosa LOMBARDI (2005; 2006a; 2006b; 2008), entre outras. Os avanços representados por este conjunto de contribuições foram analisados em um artigo referido anteriormente (MINELLA, 2013b).

Pode-se dizer que o enfoque pretendido se aproxima ainda de vários estudos mais recentes sobre a questão da feminização das profissões em várias carreiras, tais como: odontologia (Simone de Melo COSTA; Sarah Jane Alves DURÃES; Mauro Henrique Nogueira Guimarães de ABREU, 2010); ciência e tecnologia (Gilda OLINTO, 2011 ; Alice Rangel de Paiva ABREU; Maria Coleta F. A. OLIVEIRA; Joice M. VIEIRA; Gláucia S. MARCONDES, 2014);

\footnotetext{
${ }^{3}$ Este trabalho dá continuidade a estudos anteriores e integra um projeto em andamento, iniciado em março de 2015, com previsão de conclusão em fevereiro de 2018. Financiamento: CNPq

${ }^{4}$ A partir dos debates que têm ocorrido no âmbito da História das Ciências, destaca-se o artigo de Maria Margaret Lopes (2006), intitulado "Sobre convenções em torno de argumentos de autoridade". Esta autora explora as especificidades epistemológicas das áreas denominadas gênero e ciências, gênero em ciências e estudos feministas da ciência.
}

1114 Estudos Feministas, Florianópolis, 25(3): $1111-1128$, setembro-dezembro/2017 
advocacia e magistratura (Renné Martins BARBALHO, 2008; Maria da Glória BONELLI, 2013); várias carreiras de ensino superior (Maria Helena Santana CRUZ, 2012); física (Betina Stefanelo LIMA, 2013), além da coletânea organizada por Silvia Cristina YANNOULAS (2013), que inclui vários textos sobre a feminização das profissões e ocupações.

A visão sobre o perfil atual dos/as estudantes da graduação em Medicina no Nordeste e no Sul do país se ampara também na análise da literatura específica sobre mulheres na medicina, apresentada em estudo anterior sobre as trajetórias das pioneiras (MINELLA, 2015), segundo pesquisas realizadas por Maria Lúcia de Barros MOTT (1994; 1998; 2005) e Mott et al. (2008), sobre Madame Durocher e Marie Renotte; por Elisabeth Juliska RAGO (2007), a respeito da médica baiana Francisca Praguer Froés; lole Macedo VANIN (2008), sobre as primeiras médicas, farmacêuticas e odontólogas formadas pela Faculdade de Medicina da Bahia entre 1879 e 1949 e ainda análises de Monica R. SCHPUN (1999; 2010) acerca da carreira profissional e política da médica e deputada federal Carlota Pereira de Queiróz. A partir do resgate de variadas e ricas fontes históricas, essas autoras revelaram as peculiaridades do horizonte histórico enfrentado pelas pioneiras, cuja entrada na área se deu entre os finais do século XIX e o início do século XX.

No caso de pesquisas voltadas para períodos mais recentes, este estudo leva em conta valiosos escritos, como os de Léa CAMILLO-COURA, Célia Lúcia Monteiro CASTRO e Lúcia CAMILLO-COURA (1995) a respeito das percepções das médicas sobre a discriminação profissional; da coletânea intitulada Os médicos no Brasil: um retrato da realidade, organizada por Maria Helena MACHADO (1997); de Cristina BRUSCHINI e LOMBARDI (1999), sobre mulheres 'em carreiras de prestígio'; do já citado estudo de Fanny Tabak (2002), sobre a situação das mulheres em várias categorias profissionais, incluindo a medicina; de Hildete Pereira de Melo e Maria Carolina Pereira Casemiro (2004) acerca das desigualdadesde gênero na composição da Academia Nacional de Medicina e a Academia Brasileira de Ciências; de Tania Steren dos SANTOS (2004) sobre as interferências do gênero e das gerações nas percepções das médicas sobre preconceitos e discriminações e as relações entre a vida doméstica e a profissional; de Luiz Roberto MILLAN (2005) a respeito das relações entre a vocação médica e o gênero junto aos alunos da Faculdade de Medicina da Universidade de São Paulo.

Finalmente, ressaltamos que o enfoque privilegia as interseções do gênero com outros marcadores da diferença - classe, raça/etnia e gerações - de modo a refletir sobre o acesso das mulheres à carreira médica. Autoras como Kimberlé CREENSHAW (2002), Avtar BRAH (2006), Verena STOLCKE (2006) e Adriana PISCITELLI (2008) contribuem para uma compreensão das sobreposições entre os sistemas discriminatórios, suas interações e os distintos tipos de opressão que representam e que potencializam uns aos outros, multiplicando desvantagens.

\section{Abordagem e procedimentos: limites e possibilidades}

Apresentamos e discutimos aqui os dados obtidos nas instituições pesquisadas, procurando identificar semelhanças e diferenças entre os procedimentos adotados nas instituições das redes pública e privada. Na Bahia eram, em 2012, cinco cursos funcionando nas seguintes instituições: a) Faculdade de Medicina da Bahia (FMB/Universidade Federal da Bahia), criado em 1832; b) Escola Bahiana de Medicina e Saúde Pública, nascida em 1952; c) Universidade Estadual de Santa Cruz/UESC, 2000; d) Universidade Estadual de Feira de Santana/UEFS, 2003; e) Faculdade de Ciência e Tecnologia/FCT, fundada em 2004.

Em Santa Catarina, os cursos encontrados foram criados em período mais recente. entre 1960 e 2005, instalados nas seguintes instituições: a) Universidade Federal de Santa 
Catarina (UFSC), 1960; b) Universidade do Sul de Santa Catarina (UNISUL), Campus da Grande Florianópolis, Unidade Pedra Branca, 1998; c) Universidade do Sul de Santa Catarina (UNISUL), Campus Tubarão, 1998; d) Universidade de Joinville (UNIVILLE), 1998; e) Graduação em Medicina da Universidade do Oeste de Santa Catarina (UNOESC), Campus de São Miguel do Oeste, 2001 ; f) Universidade Comunitária da Região de Chapecó (UNOCHAPECÓ), 2005; g) Universidade de Blumenau (FURB), 1989; h) Universidade do Extremo Sul Catarinense (UNESC), 1998; i) Universidade do Planalto Catarinense (UNIPLAC), 2003; j) Universidade do Vale do Itajaí (UNIVALI), 1998.

Até o momento, a abordagem tem sido quantitativa, mas nas etapas posteriores está prevista a realização de entrevistas, no intuito de indagar sobre as interferências de gênero na escolha da carreira e das especialidades médicas, principalmente entre estudantes cotistas. Entre as instituições da rede pública, foram selecionadas a Universidade Federal da Bahia (UFBA) e a Universidade Federal de Santa Catarina (UFSC). Informações sobre 1.259 e 805 estudantes foram sistematizadas, respectivamente, sendo obtidas através de questionários socioeconômicos aplicados durante a inscrição nos exames vestibulares de cada instituição. Foram selecionadas somente as variáveis sexo, faixa etária, renda familiar, cor/etnia e origem escolar dos aprovados e classificados entre 2005 e 2012 . O levantamento foi realizado junto às pró-reitorias e aos núcleos de processamento de dados, entre os anos de 2012 e 2014, por meio de consulta aos vários relatórios disponíveis nos sites dessas instituições. Entretanto, não foi possível desagregar as variáveis de forma a considerar como independente a primeira delas (sexo); por consequência, as categorias mulheres e homens não foram separadas nas discussões sobre idade, renda, origem escolar e cor/ etnia. Avaliamos que, apesar desse limite, os dados obtidos contribuem para o entendimento geral do perfil dos/as estudantes e para uma compreensão do processo de feminização da área.

Quanto às instituições privadas, várias dificuldades foram enfrentadas, desde a falta de respostas às nossas insistentes solicitações até a carência de informações minimamente sistematizadas. Depois de várias tentativas, as universidades com cursos de medicina previamente selecionadas - Universidade de Joinville (UNIVILLE), em Santa Catarina, e Universidade Estadual de Santa Cruz (UESC), na Bahia - foram substituídas, acrescentando-se outras instituições. Assim, em Santa Catarina foram incluídas a Universidade do Vale do Itajaí (UNIVALI) e a Universidade do Extremo Sul Catarinense (UNESC), campus Criciúma. No estado da Bahia, a Escola Bahiana de Medicina e Saúde Pública (EBMSP) e a Faculdade de Tecnologia e Ciências (FTC). Os segmentos abordados também foram redefinidos a partir dos dados aos quais tivemos acesso. O levantamento de dados nessas instituições iniciou em 2013 e se estendeu até o segundo semestre de $2015 .^{5}$

Na EBMSP, foram sistematizados dados sobre sexo, idade, renda e origem escolar de 1.300 estudantes classificados no exame seletivo entre 2006 e o primeiro semestre de 2013. Não encontramos informações sobre cor nem os dados relativos ao ano de 2009. No caso da FTC, na impossibilidade de acessar as informações sobre aprovados/as e classificados/ as no exame vestibular, foram sistematizados os dados dos/as 32.536 inscritos/as no curso entre 2009 e 2013. Não encontramos registros sobre cor e/ou pertencimento étnico, nem sobre origem escolar. Em Santa Catarina, obtivemos os dados sobre sexo, idade e cor dos/ as 476 egressos/as do curso da UNIVALI, entre 2005 e 2012, não tendo sido encontradas

${ }^{5}$ Contribuíram no levantamento dos dados secundários as/os seguintes estudantes: Maria Clara Barbosa Oliveira Casagrande, na UNIVALI; Sabrina Guerra Guimarães, na UFBA, na FTC e na EBMSP; Zuleika Rodrigues, na UFBA e na UNESC. Pietro Volpani se encarregou de parte significativa da sistematização dos dados gerais sobre UFBA e UFSC.

1116 Estudos Feministas, Florianópolis, 25(3): $1111-1128$, setembro-dezembro/2017 
informações sobre renda e origem escolar; esta instituição parece não contar com informações sistemáticas sobre os/as classificados/as. No caso da UNESC, foram levantados os dados sobre sexo, idade e origem escolar dos/as 519 classificados/as entre 2010 e 2015. A instituição não sistematiza dados sobre renda familiar e cor/etnia dos/as estudantes.

De um modo geral, portanto, os dados sobre o perfil dos/as estudantes nas instituições públicas estão mais completos. Os dados gerais foram sintetizados a partir das informações disponíveis, sendo considerados segmentos distintos e uma pequena diferenciação nos períodos (iguais em três instituições e ligeiramente distintos em três delas).

Têm-se, portanto, seis instituições, duas públicas federais (uma em cada estado) e quatro privadas, sendo duas em cada estado. Os dados da UFSC, da UFBA, da EBMSP e da UNESC dizem respeito aos/às estudantes que foram aprovados/as e classificados/as nos exames seletivos. Os dados da FTC se referem aos inscritos/as no vestibular e os da UNIVALI aos/às egressos/as que se formaram. Quanto aos períodos, houve uma coincidência nos casos da UFSC, UFBA e UNIVALI, de 2005 a 2012; na FTC, encontramos informações de 2009 a 2013.1; na Bahiana, de 2006 a 2013.1, faltando apenas o ano de 2009. Em todas as instituições foi possível obter informações sobre sexo e idade. Em três instituições privadas (FTC, EBMSP e UNESC) a falta de registros institucionais sobre a cor talvez possa ser explicada pela inexistência de cotas - fato que, no entanto, não ocorreu na UNIVALI, onde conseguimos informações sobre a cor daqueles/as que se formaram.

Ressaltamos ainda que a revisão da literatura tem se dado paralelamente aos demais procedimentos da pesquisa, focalizando desde a trajetória das pioneiras no campo da medicina no Brasil até um balanço dos temas abordados e das metodologias utilizadas em diferentes estudos sobre as relações entre gênero e ciências, com o objetivo de identificar suas lacunas e interrogar até que ponto as interseções gênero, classes, gerações e etnia têm sido contempladas (MINELLA, 2013a; 2013b).

\section{O perfil nas redes pública e privada: semelhanças e diferenças}

Do ponto de vista interseccional, os resultados do levantamento sinalizam que o perfil dos/as estudantes da rede pública se caracteriza pela crescente participação das mulheres, pela presença majoritária de jovens, por uma maior diversidade de pertencimento aos estratos socioeconômicos, aliada aos avanços no quesito cor, estes últimos mais expressivos na UFBA. Na rede privada, as duas primeiras características também foram constatadas, mas há diferenças em relação às demais. Na UFBA, entre os/as 1.259 classificados/as no curso entre 2005 e 2012, para uma média de 160 vagas, a maioria foi do sexo masculino - 58,9\% contra $41,1 \%$ de mulheres. ${ }^{6}$ A UFSC apresenta proporções semelhantes; para uma média de 100 vagas por ano prevaleceram também os homens $(55,29 \%)$, contra um número significativo de mulheres $(44,71 \%)$.

Tanto na Bahia quanto em Santa Catarina a tendência à feminização da rede privada se assemelha à da rede pública, com ligeiras variações. Na FTC, entre os/as inscritos/as no exame vestibular, as mulheres também constituíram maioria, ou seja, 20.447, correspondendo a $62,8 \%$, enquanto os homens representaram 12.089 , totalizando $37,16 \%$; em 2009, inscreveram-se 2.068 mulheres (61,0\%), contra 1.369 homens (39,0\%); em 2013, a proporção de mulheres que realizaram a inscrição aumentou, sendo $4.262(66,8 \%)$ contra $2.178(33,2 \%)$ homens. Na EBMSP, dos 1.300 aprovados entre 2006 e 2013.1 , embora faltem

${ }^{6}$ Vale ressaltar que a proporção de homens foi mais alta no curso de Medicina da UFBA do que aquela registrada como média em todas as áreas entre 2005 e 2008: homens, 50,6\% e mulheres, 49,4\% (UFBA, 2008). 
as informações sobre o ano de 2009, é possível observar a mesma tendência: o número de mulheres chegou a 728 (56,0\%); enquanto os homens atingiram $547(42,1 \%)$; vinte e cinco estudantes não declararam o sexo, chegando a representar $1,92 \%$.

Em Santa Catarina, observou-se tendência semelhante: entre os/as classificados/as da UNESC entre 2010 a 2015, tem-se 59,8 homens contra 40,2\% mulheres. Na UNIVALI, dos/ as 476 formados/as entre 2005 e 2012, as mulheres também prevaleceram, totalizando 240 $(50,4 \%)$ sobre 236 homens $(49,6 \%)$. Observando a evolução ao longo dos anos, constatamos que, em 2005, apenas 15 mulheres contra 20 homens concluíram o curso; já em 2012, o número delas subiu para 30 e o deles para 31.

Os estudos de June HAHNER (2003), Elizabeth Rago (2007) e Monica Schpun (1999; 2010) revelam que as pioneiras da medicina no Brasil foram uma exceção em turmas majoritariamente constituídas por homens. Também ressaltam que elas atuaram nas áreas de Ginecologia, Obstetrícia e Pediatria, tal como a maioria daquelas que entraram na academia nas décadas subsequentes, configurando um nicho profissional.

Como demonstra José TAVARES-NETO (2008), entre os anos 1812 - quando a primeira turma da Faculdade de Medicina da Bahia concluiu seus estudos - e 1912, ou seja, no espaço de um século, 2.691 homens se diplomaram contra apenas sete mulheres $(0,26 \%)$. Aproximadamente um século depois, entre 1913 e 2008, embora a presença delas tenha se tornado numericamente significativa a partir da década de 50 , tem-se um total de 7.518 homens titulados contra 2.731 mulheres $(73,45$ contra $26,6 \%)$, segundo o pesquisador. Considerando o total de diplomados/as ao longo de dois séculos, tem-se, portanto, 10.209 homens e 2.738 mulheres (12.947 no total geral, sendo $78,8 \%$ homens contra $21,2 \%$ de mulheres). Tais números são importantes justamente porque ressaltam a forte assimetria de gênero que se instalou no período.

Vanin (2008) ressalta que, na Bahia, o incremento da participação das mulheres deu-se mais nos cursos de Farmácia e Odontologia e menos no curso de Medicina, pois entre os anos de 1879 e 1949 (quando esses três cursos eram ofertados pela Faculdade de Medicina) formaram-se 168 mulheres em Farmácia, 160 em Odontologia e 84 em Medicina. A autora assinala que havia mais mulheres naquelas áreas menos prestigiadas, ligadas ao atendimento às próprias mulheres e às crianças.

Maria Lúcia Mott et al. (2008) analisam o perfil de médicos e médicas que se diplomaram em São Paulo entre os anos de 1892 e 1932, mediante consulta aos dados disponíveis no Centro de Memória da Saúde Pública de São Paulo, concluindo que o número de médicas registrado no período equivalia a apenas $1,0 \%$. Essa proporção se manteve durante décadas no país. Maria Helena Machado (1997) sinaliza que o processo de feminização da carreira teria se iniciado depois da metade do século XX, mostrando que, se em 1940 o contingente feminino equivalia a apenas 1,0\%, na década de 1990 a proporção tinha atingido $35,0 \%$ - sendo que no segmento com menos de trinta anos as mulheres representavam 50,0\%. Também Cristina Bruschini e Maria Rosa Lombardi (1999), já citadas neste artigo, encontram percentual semelhante $(38,0 \%)$ nesse último período, quando analisam a presença das mulheres 'em carreiras de prestígio', ou seja, atuantes nas áreas de Medicina, Arquitetura, Direito e Engenharia, ponderando inclusive diversos aspectos relativos às especificidades desse processo.

Estudos mais recentes feitos no Brasil, por exemplo, evidenciam a intensificação da feminização do campo analisado. Ao observar a evolução da população de médicos segundo o sexo, a partir de uma ampla base de dados, Scheffer e Cassenote (2013, p. 270) assinalam que "as mulheres aparecem com 22,0\% e 21,0\% nos anos de 1910 e 1920, em relação ao total". O percentual recua para 19,10\% em 1930 e cai mais ainda nos anos posteriores, atingindo 12,99\% em 1960. Mas a partir de 1970 é possível observar que o 
percentual sobe "paulatinamente para 23,47\% em 1980; 30,80\% em 1990; 35,82\% em 2000; até atingir $39,91 \%$ em 2010" (2013, p. 270).

Conforme assinalamos no início deste artigo, esta tendência nacional reflete o panorama internacional. Riska (2001) afirma que, em 1950, a proporção de mulheres na profissão médica diferia muito de um país para o outro: nos Estados Unidos 6\% eram mulheres; na União Soviética, 77\%; na Finlândia, 21\%. Nos demais países escandinavos (Dinamarca, Noruega e Suécia), em torno de 10,0\%. Cinquenta anos depois, a situação na Rússia se manteve, nos países escandinavos há um equilíbrio e nos EUA as mulheres alcançaram $25 \%$. Diante desse incremento, a autora levanta algumas questões relevantes que, sem dúvida, merecem estudos específicos e análises comparativas: as mulheres estão mudando a medicina? A medicina tem se transformado num trabalho para mulheres? As políticas da área estão preparadas para absorver essa mudança e promover os ajustes necessários? (RISKA, 2001).

Com relação à idade, observamos que as instituições trabalham com intervalos ligeiramente distintos. Evitamos uma padronização para manter uma fidelidade aos seus critérios, considerando que devem refletir, de alguma forma, peculiaridades regionais. Os dados levantados, tanto na rede pública quanto na privada, mostram a predominância de uma população jovem, confirmando as tendências observadas no país, segundo o Censo de Educação Superior (INEP, 2013).

Na UFBA, rede pública da Bahia, a faixa entre 17 e 19 anos representa $60,7 \%$, destacando-se no período considerado; entre 20 e 25 anos, o percentual é significativo, atingindo $31,76 \%$; a partir dos 25 anos poucos foram os que ingressaram, registrando-se apenas 6,04\% entre 25 e 47 anos e mais. Do ponto de vista geracional, observamos um perfil jovem também entre os anos de 2005 e 2008 em todos os cursos da área das Ciências Biológicas e Profissões de Saúde, quando os/as inscritos/as atingiram 77,0\% a 85,0\%, configurando os maiores percentuais entre todas as áreas (UFBA, 2008, p. 20). Os dados da UFSC mostram proporções semelhantes: a maior parte se concentra na faixa entre 19 a 24 anos, correspondendo a $77,86 \%$; até 18 anos, eram 13,11\%, com significativo declínio a partir dos 25 anos. Entre esse limite e os 29 anos, tem-se apenas 6,61\% de estudantes; entre 30 a 34 anos, $1,65 \%$ e, finalmente, acima dos 35 anos, apenas $0,77 \%$.

Na rede privada, especialmente na FTC e na EBMSP, observamos uma concentração maior na faixa até 18 anos. Entre os/as inscritos/as na FTC, tem-se 15.949, ou seja, uma maioria com até 18 anos, representando 49,2\%; na faixa seguinte, entre 19 e 25 anos, há também um número expressivo: 10.991 (33,8\%); apenas $17,2 \%$ do total se concentram acima dos 26 anos, sendo que apenas $3,0 \%$ tinha 40 anos e mais. Na EBMSP também constatamos uma alta concentração até os 20 anos de idade: 1.090 estudantes, correspondendo a 83,9\%; entre 21 e 24 anos há uma queda expressiva: 141 estudantes, ou seja, 10,8\%; entre 25 e 40 anos registraram-se apenas 43 estudantes, isto é, 3,3\%, e ninguém acima dos quarenta anos.

Na rede privada de Santa Catarina a mesma tendência foi constatada. Na UNESC, cerca de 17,0\% têm entre 18 e 20 anos, a maioria se concentra entre 21 e $2560,0 \%$ e apenas $14,4 \%$ encontram-se entre 26 e 30 anos. A partir desse limite, as frequências mostramse rarefeitas: entre os 476 formados/as da UNIVALI, 292 deles/as concluíram o curso entre 23 e 25 anos, ou seja, uma maioria que representa 61,3\%; em segundo lugar, tem-se a faixa entre 26 e 28 anos, representativa de 147 formados/as (30,9\%); entre os 29 e os 40 anos, o percentual atingiu apenas $6,7 \%$, e não houve formados acima de 41 anos.

A diplomação em torno dos vinte e poucos anos se mantém na área, sendo frequente também entre as pioneiras. Rago (2007) mostra, por exemplo, que Francisca Praguer Froés se formou em 1893, aos 21 anos, e Hahner (2003) esclarece que Rita Lobato se formou aos 
21 anos e Ermelinda Lopes Vasconcelos aos 22 anos. Segundo os resultados da Pesquisa Nacional por Amostra de Domicílios Contínua (PNAD), o percentual de estudantes que frequentavam a educação superior representava cerca de $30,0 \%$ da população do Brasil na faixa etária de 18 a 24 anos em 2012 (PNAD/IBGE, 2012). ${ }^{7}$

Quanto à origem escolar, no caso da rede pública, constatamos diferenças regionais. Na UFBA, prevaleceram as escolas privadas $(54,5 \%$ contra $45,5 \%$ das públicas) em todo o período considerado. Na UFSC, os dados do vestibular de 2012 mostram que também houve uma prevalência maior das escolas privadas $(70,0 \%$ contra $30,0 \%$ das públicas). Certamente nos períodos anteriores à implantação do Programa de Ações Afirmativas (PAA), o predomínio das escolas privadas era maior. Os dados deste Programa na UFSC evidenciam que, antes da sua implantação, entre 2004 e 2007, o percentual médio de classificados/as oriundos/as de escolas públicas equivalia a apenas 18,1\%. Entre 2008 e 2012, o percentual médio passou para $32,1 \%$. Portanto, no caso da UFSC, o percentual daquelas/es que realizaram o ensino secundário nas escolas públicas e que ingressaram em 2012 ficou dentro da média. Além de outras peculiaridades socioculturais e econômicas regionais, as diferenças certamente se explicam também devido à implantação do PAA na UFBA ter antecedido em três anos à da UFSC. No caso da rede privada baiana, não encontramos os dados da FTC sobre origem escolar. Já na EBMSP, prevaleceu nitidamente a escola particular, representando $92,0 \%$. O mesmo ocorreu na UNESC, onde se encontrou uma maioria que estudou em escolas privadas $(85,8 \%)$, contra apenas $14,2 \%$ que frequentaram escolas públicas. Em Santa Catarina, na UNIVALI, também não foram encontrados registros a esse respeito.

No que se refere à renda familiar na rede pública, predominou, tanto na UFBA quanto na UFSC, a faixa entre cinco a dez salários mínimos (25,4\% e $31,8 \%$, respectivamente). No caso da UFBA, esta faixa pode ser considerada alta, em comparação com outros cursos, pois entre 2005 e 2008 os dados gerais mostram que 45,2\% dos classificados/as situavamse no intervalo inferior a cinco salários mínimos. Em seguida, encontram-se os intervalos correspondentes a três a cinco, sendo $18,4 \%$ na UFBA e $14,8 \%$ na UFSC. Uma concentração maior nas faixas mais altas foi encontrada na UFSC: $24,3 \%$ dos estudantes declararam uma renda familiar entre dez a vinte salários mínimos, contra 18,1\% da UFBA.

Apesar da alta concorrência - que chegou a 39 candidatos por vaga em 2011 e 49 em 2012 na UFBA - observamos que, nessa instituição, entre 2,05 e 10,9\% dos/as estudantes provêm de famílias cuja renda correspondia a um salário e entre um e três salários mínimos, respectivamente. Na UFSC, as proporções dessas faixas foram menores, correspondendo a 0,55 e $7,60 \%$. No entanto, segundo as informações sobre a implantação do PAA nessa instituição, se antes de 2008 40\% dos/as classificados/as declararam serem provenientes de famílias com renda entre dez a vinte e de sete a dez salários mínimos (de acordo com dados de 2012), após 2008 as faixas de um a três e de três a cinco salários mínimos passaram a ocupar $47 \%$ das vagas. ${ }^{8}$

Observa-se, portanto, um perfil mais diversificado na UFBA do que na UFSC e, em ambas, o sistema de cotas tem contribuído significativamente para esse resultado também em termos mais gerais. No caso da UFBA, além da Medicina, em outras carreiras dentre as mais concorridas (Psicologia, Direito, Comunicação com habilitação em Jornalismo e

\footnotetext{
${ }^{7}$ Dados detalhados sobre juventude e ensino superior (incluindo idade versus cor) em: Marcos Roberto MESQUITA; Gessé MARQUES JÚNIOR; Anderson Azevedo SIMÕES (2012).

${ }^{8}$ Essa maior diversificação do perfil socioeconômico (e também étnico) tenderá a se ampliar, tendo em vista as políticas de inclusẫo. No vestibular de 2013 , a UFSC aumentou de 30 para $35,0 \%$ o ingresso por PAA (25,0\% das vagas para egressos de escolas públicas e $10,0 \%$ para candidatos/as negros/as).
} 
Enfermagem), os percentuais das famílias com renda mensal entre um a cinco salários mínimos também foram altos (UFBA, 2008, p. 29).

Embora estes dados não estejam desagregados por sexo, assinala-se as diferenças em relação ao perfil das pioneiras, que ingressaram na área entre os finais do século XIX e as primeiras décadas do século XX. Conforme a literatura citada, em geral elas provinham de famílias da elite ou das camadas médias, setores que, embora tivessem, em alguns casos, vínculos originários com o meio rural, se estabeleceram nas grandes cidades. Também era comum que, entre esses segmentos, se encontrassem filhos e filhas de migrantes europeus - situação que provavelmente se prolongou até poucas décadas atrás.

As camadas médias e alta prevalecem claramente na rede privada baiana. Na FTC, houve a prevalência de renda mensal familiar acima de $\mathrm{R} \$ 10.500,00(30,66 \%)$, seguida de entre R\$7.000,00 a 10.500,00 (14,16\%); apenas 1.293 inscritos/as declararam renda familiar de até $\mathrm{R} \$ 700,00$, representando $4,06 \%$. Na EBMSP, a faixa acima de trinta salários mínimos foi majoritária, num total de 253 (19,5\%). No cômputo geral, considerando todas as faixas nesta última, predominou claramente a renda acima de dez salários mínimos (72,3\%); apenas 6 estudantes declararam entre 1 a 2 salários mínimos. Na UNIVALI e na UNESC não foram encontrados dados sobre renda.

Quanto ao quesito cor, observamos claramente as interferências dos perfis étnicos regionais na rede pública. Na UFBA, a maior parte se declarou parda $(53,4 \%)$; em seguida, prevaleceu a cor branca $(24,1 \%)$ e a preta $(8,49)$. Pardos e pretos predominam, portanto, atingindo $61,8 \%$. Indígenas e amarelos representam os menores contingentes, com 2,0 e $1,1 \%$, respectivamente. Não obstante a pequena proporção destes últimos, a diversidade se explica tanto em razão do amplo contingente de afrodescendentes no estado da Bahia, estimado em cerca de $73,0 \%$ em 2009 pela PNAD (IBGE, 2009), quanto pela implantação do sistema de cotas e ainda pela criação do Programa Permanecer, no ano de 2007, que visa à consolidação das políticas de ações afirmativas (UFBA, 2008, p. 4) ${ }^{9}$. Ainda segundo dados da UFBA:

[...] os cursos nos quais o ingresso dos candidatos de cor preta - e mesmo dos pardos era bem inferior em relação aos brancos em períodos anteriores, notadamente aqueles que conferem um maior status social e econômico, apresentam mudanças consideráveis. Em cursos como Medicina, Direito e Odontologia, a representação de pretos e pardos foi visivelmente maior (Medicina 72,5\%; Direito 69,7\% e Odontologia 72,6\%) (UFBA, 2008, p. 20).

Já na UFSC, instituição cujos estudantes são originários principalmente do próprio estado de Santa Catarina, do Rio Grande do Sul e do Paraná, predominaram claramente os brancos $(82,9 \%)$, enquanto que os pardos representaram $9,6 \%$ e os pretos apenas $3,7 \%$. Esta predominância era esperada de certa forma, haja vista que são de regiões nas quais significativos contingentes de imigrantes de origens italiana e alemã se estabeleceram nos meios rurais e urbanos, e onde a proporção de negros é bem menor do que no Nordeste. Segundo o IBGE, a proporção de autodeclarados brancos em Santa Catarina atingiu 84,0\% em 2010. A articulação do perfil étnico com a condição de classe é evidente, dado que na UFSC as faixas de rendimento foram mais altas do que na UFBA. De qualquer forma, os avanços na primeira foram também consideráveis. Os dados sobre o quesito cor na UFBA e na UFSC confirmam os resultados das pesquisas do IBGE (2010) sobre o aumento de pretos e pardos no ensino superior no país entre 2000 e 2010.

${ }^{9} \mathrm{Em} 2005$, quinze universidades públicas (federais e estaduais) tinham adotado políticas de ação afirmativa Entre elas, as pioneiras dessas ações: Universidade Estadual do Rio de Janeiro, Universidade Estadual do Norte Fluminense, Universidade do Estado da Bahia e a Universidade de Brasília. 
De acordo com o Relatório de Avaliação do PAA da UFSC, elaborado pela Comissão Institucional de Acompanhamento e Avaliação, houve um aumento considerável no percentual de pretos a partir de 2008: "De 2004 a 2007, eles eram 1,0\% dos ingressantes, $e$ passaram a ser $4,6 \%$. Os pardos aumentaram de 7,5\% (de 2004-2007) para $8,7 \%$ (20082012). Os negros passaram de $8,5 \%$ para $13,3 \%$ de antes para depois do PAA" (UFSC, 2012 , p. 11). A Comissão conclui que houve um aumento de $56 \%$ no percentual de negros do PAA, configurando um aumento significativo, bem como tem constatado que estudantes negros são os que apresentam a menor taxa de evasão, contrariando as expectativas dos opositores às políticas de cotas.

As vagas por cotas têm sido preenchidas na Medicina da UFBA com base nas normas sobre reservas de vagas da Resolução $n^{\circ} 01 / 04$, do Conselho de Ensino, Pesquisa e Extensão, aprovada em julho de $2004 .^{10}$ No caso da UFSC, as reservas são definidas atualmente pela Resolução $n^{\circ} 26$, aprovada pelo Conselho Universitário em outubro de 2012. Este documento ajusta as normas anteriormente vigentes à Lei Resolução $n^{\circ} 12.711$ e outras deliberações do governo federal.

Infelizmente, na rede privada baiana (FTC e EBMSP) não constam dados sobre cor. Em Santa Catarina, na UNESC tampouco foram encontradas informações sobre este quesito, enquanto na UNIVALI, entre os formados, a maioria (447) se declarou branca, chegando a $93,9 \% ; 18$ se declararam pardos (3,78\%); 7 , amarelos ( $1,47 \%$ ). Apenas 3 , ou seja, $0,63 \%$, declararam-se pretos e apenas um, indígena.

\section{Considerações finais}

Entre os limites da pesquisa, destacamos primeiro a escassez de informações sobre a rede privada. Sem dúvida, houve um avanço bem maior no levantamento na rede pública nos dois Estados. Novas tentativas estão sendo feitas com vistas a estabelecer uma visão mais completa sobre o perfil da rede privada nas próximas etapas da pesquisa. Segundo, em virtude das dificuldades e peculiaridades dos bancos de dados das instituições da rede privada, se tornou necessário trabalhar com segmentos distintos da população estudantil (classificados/as nos exames de seleção na EBMSP e da UNESC; inscritos/as nos exames de seleção, na FTC; formados/as e egressos/as, na UNIVALI) e houve também uma pequena diferenciação nos períodos, iguais em três instituições (2005 a 2012) e ligeiramente distintos em três delas (2006 a 2013; 2009 a 2013.1; 2010 a 2015.1).

Não obstante tais limites, avaliamos que os resultados obtidos permitem uma compreensão geral das diferenças e semelhanças do perfil das/os estudantes em três âmbitos: a) em instituições públicas e privadas; b) nas mais tradicionais - as duas públicas - e nas mais modernas - privadas; c) nas capitais e no interior de um dos Estados, favorecendo as reflexões sobre as possibilidades de absorção das mulheres e de estudantes não brancos, não jovens e de baixa renda. Permitem também uma confirmação e atualização de informações apontadas em várias das pesquisas referidas no início desse artigo.

Sintetizando, os resultados mostram que o perfil dos/as estudantes nas redes pública e privada tem se caracterizado pela expansão da participação das mulheres e pela presença majoritária de jovens entre 17 e 25 anos, confirmando as tendências do país segundo o Censo de Educação Superior (INEP, 2013). Foram constatadas diferenças regionais quanto à origem escolar na rede pública e também diferenças entre as redes. Tanto na UFBA quanto na UFSC predominaram as escolas privadas, sendo que na primeira

\footnotetext{
${ }^{10}$ A Resolução na íntegra consta no Manual do Candidato ao Vestibular da UFBA (2013) e no site www.vestibular.ufba.br.
} 
numa proporção menor. Na EBMSP e na UNESC, como se esperava em instituições privadas, predominou claramente a escola particular com $92,0 \%$ e $85,8 \%$, respectivamente. Na FTC e na UNIVALI não foram encontrados registros.

Em termos socioeconômicos, identificamos um perfil mais diversificado nas instituições públicas, principalmente na UFBA. Nelas, foi mais frequente a presença de estudantes que declararam renda familiar entre $\mathrm{R} \$ 5.000,00$ e $\mathrm{R} \$ 10.000,00$. Consideramos que, em ambas, o sistema de cotas tem contribuído significativamente para esse resultado. No caso da UFBA, além da Medicina, em outras carreiras entre as mais concorridas (Psicologia, Direito, Comunicação com habilitação em Jornalismo e Enfermagem), os percentuais das famílias com renda mensal entre 1 a 5 salários mínimos também foram altos (UFBA, 2008, p. 29). Faixas de renda mais alta predominaram claramente na rede privada baiana, pois a maioria afirmou que a renda familiar ultrapassava $\mathrm{R} \$ 10.000,00$ - tanto na EBMSP quanto na FTC.

No que se refere à cor, no caso da rede pública, também foram observadas diferenças que podem ser atribuídas às interferências dos perfis étnicos por região: a maior parte se declarou parda na UFBA e branca na UFSC. Em termos gerais, a proporção de autodeclarados pretos revela uma tendência ascendente; a dos descendentes dos índios mostra também um incremento, embora estes continuem representando uma minoria. $O$ perfil étnico da rede pública, juntamente com a diversidade das faixas de renda das famílias de origem, aponta para a democratização das universidades públicas federais. Embora os homens continuem sendo maioria, os dados sinalizam avanços no sentido da equidade de gênero que, sem dúvida, tem ocorrido não apenas na área da Medicina, mas no campo acadêmico de modo geral.

No entanto, há limites que precisam ser superados. Por exemplo, do ponto de vista geracional, a absorção tanto de homens quanto de mulheres não jovens, ou seja, com trinta anos ou mais, é mínima. Os patamares de renda revelam que, no sul, o pertencimento aos estratos socioeconômicos mais elevados é mais pronunciado. Na UFSC, os percentuais de pretos e pardos continuam baixos, mesmo com a implantação do regime de cotas. Uma análise preliminar do perfil étnico na rede privada ficou prejudicada pela carência de informações nos bancos de dados das instituições. Quanto aos egressos da UNIVALI, a grande maioria $(93,9 \%)$ se declarou branca.

Consideramos que a revisão da literatura, bem como os dados aqui apresentados, estimula a continuidade desse estudo. Posteriormente, prosseguiremos com o levantamento de dados quantitativos sobre o perfil dos/as estudantes, com ênfase na rede privada. Mediante procedimentos qualitativos, pretendemos também analisar o impacto das políicas de ações afirmativas na rede pública, dialogando com estudantes cotistas sobre suas trajetórias, sondando as interferências de gênero na escolha das especialidades médicas e verificando permanências e mudanças em relação aos resultados de pesquisas anteriores.

\section{Referências}

ABREU, Alice Rangel de Paiva; OLIVEIRA, Maria Coleta F. A.; VIEIRA, Joice M.; MARCONDES, Gláucia S. (Orgs.). "Equidade de Gênero na Sociedade do Conhecimento no Brasil: presença feminina na ciência e tecnologia". Document de Travail du Mage, v. 18, p. 93-120, 2014.

AZEVEDO, Nara; FERREIRA, Luiz Otávio. "Modernização, políticas públicas e sistema de gênero no Brasil: educação e profissionalização feminina entre as décadas de 1920-1940". Cadernos Pagu, Campinas, Unicamp, Núcleo de Estudos de Gênero, p. 217-254, 2006. 
BANCO MUNDIAL. Relatório sobre o Desenvolvimento Mundial de 2012. Igualdade de Gênero e Desenvolvimento, 2011 . Disponível em: http://siteresources.worldbank.org/INTWDR2012/ Resources/7778105-1299699968583/7786210-1315936231894/OverviewPortuguese.pdf. Acesso em: 30/04/2015.

BARBALHO, Renné Martins. A feminização das carreiras jurídicas: construções identitárias de advogados e juízes no âmbito do profissionalismo. 2008. Tese (Doutorado em Sociologia), Centro de Educação e Ciências Humanas, Universidade Federal de São Carlos, São Paulo.

BONELLI, Maria da Glória. "Profissionalismo, diferença e diversidade na advocacia e na magistratura paulistas". Revista Brasileira de Ciências Sociais, São Paulo, ANPOCS, v. 28, n. 83, p. 125-140, 2013.

BRAH, Avtar. "Diferença, diversidade, diferenciação". Cadernos Pagu, Campinas, n. 26, p. 329-365, 2006.

BRUSCHINI, Cristina; LOMBARDI, Maria Rosa. "Médicas, arquitetas, advogadas e engenheiras: mulheres em carreiras de prestígio". Revista Estudos Feministas, Florianópolis, v. 7, n. 1 e 2, p. 9-24, 1999.

CABRAL, Carla Giovana e BAZZO, Walter Antonio. "As mulheres nas escolas de engenharia brasileiras: história, educação e futuro". Cadernos de Gênero e Tecnologia, Curitiba, CEFET/PR, v. 1, n. 4, p. 3-9, 2005.

"Pelas telas, pela janela: o conhecimento dialogicamente situado". Cadernos Pagu, Campinas, n. 27, p. 63-97, 2006a.

"Investigando o caráter situado do conhecimento: reflexões sobre epistemologias feministas e educação científica e tecnológica". Revista Tecnologia e Sociedade, Curitiba, UTFPR, n. 3, p. 23-41, 2006b.

"Mapeando e refletindo a construção do campo disciplinar 'Estudos Feministas da ciência e da tecnologia' no Brasil nos percursos do Fazendo Gênero". In: SEMINÁRIO INTERNACIONAL FAZENDO GÊNERO 8 - CORPO, VIOLÊNCIA e PODER. Florianópolis, 25 a 28 de agosto de 2008. Anais... Florianópolis: Universidade Federal de Santa Catarina, 2008.

CAMILLO-COURA, Léa; CASTRO, Célia Lúcia Monteiro; CAMILLO-COURA, Lúcia. "Mulher médica: a discriminação profissional". Academia Nacional de Medicina, Rio de Janeiro, n. 155, v. 3, p. 135-139, Anais... julho-setembro 1995.

COSTA, Simone de Melo; DURÃES, Sarah Jane Alves; ABREU, Mauro Henrique Nogueira Guimarães de. "Feminização do curso de Odontologia da Universidade Estadual de Montes Claros". Ciência \& Saúde Coletiva, Rio de Janeiro, v. 15, suppl. 1, p. 1873-1875, 2010.

CREENSHAW, Kimberlé. "Documento para o encontro de especialistas em aspectos da discriminação racial relativos ao gênero". Revista Estudos Feministas, Florianópolis, v. 10, n. 1, p. 171-188, 2002.

CRUZ, Maria Helena Santana (Org.). Mapeando diferenças de Gênero no Ensino Superior. São Cristóvão: UFS, 2012.

FOX KELLER, Evelyn. "Making a Difference: feminist movement and feminist critiques of science". In: CREAGER, A.; LUNDBECK, E.; SCHIEBINGER, L. (Eds.). Feminism in twentiethcentury science, technology, and medicine. Chicago/London: The University of Chicago Press, 2001. p. 98-109.

; LONGINO, Helen (Eds.). Feminism and Science. Oxford; New York: Oxford University Press, 1996. (Oxford Readings in Feminism)

HARAWAY, Donna. "Um manifesto para os cyborgs: ciência, tecnologia e feminismo socialista na década de 80". In: HOLLANDA, Heloísa Buarque de (Org.). Tendências e impasses: 0 feminismo como crítica da cultura. Rio de Janeiro: ROCCO, 1994. p. 243-287. 
"Saberes localizados: a questão da ciência para o feminismo e o privilégio da perspectiva parcial". Cadernos Pagu, Campinas, n. 5, p. 07-42, 1995.

HARDING, Sandra. Ciencia y Feminismo. Madrid: MORATA, 1996.

FERREIRA, Luiz Otávio; AZEVEDO, Nara; GUEDES, Moema; CORTES, Bianca. "Institucionalização das ciências, sistema de gênero e produção científica no Brasil (1939-1969)". História, Ciências, Saúde - Manguinhos, Rio de Janeiro, Fundação Oswaldo Cruz, v. 15, p. 4371, 2008.

GUEDES, Moema de Castro. "A presença feminina nos cursos universitários e nas pósgraduações: desconstruindo a ideia de universidade como espaço masculino". História, Ciências, Saúde - Manguinhos, Rio de Janeiro, Fundação Oswaldo Cruz, v. 15, p. 117 132, 2008.

HAHNER, June. Emancipação do Sexo Feminino. A luta pelos direitos da mulher no Brasil. 1850-1940. Florianópolis: Mulheres; Santa Cruz do Sul: EDUNISC, 2003.

IBGE - INSTITUTO BRASILEIRO DE GEOGRAFIA E ESTATISTICA. Censo Demográfico 2010. Educação de Deslocamento. Resultados da Amostra 2010. Rio de Janeiro: IBGE, 2010.

Pesquisa Mensal de Emprego. Algumas características da inserção das mulheres no mercado de trabalho. Recife, Salvador, Belo Horizonte, Rio de Janeiro, São Paulo e Porto Alegre 2003-2008. Rio de Janeiro: IBGE, 2009.

Mulher no Mercado de Trabalho. Perguntas e Respostas. Rio de Janeiro: IBGE, 2012. Síntese de Indicadores Sociais. Rio de Janeiro: IBGE, 2012.

INEP - INSTITUTO NACIONAL DE ESTUDOS E PESQUISAS EDUCACIONAIS ANÍSIO TEIXEIRA. CensO de Educação Superior. Ministério da Educação, 2013. Disponível em: inep.gov.br/ educação_superior/censo_superior/apresentação/2014/coletiva_censo_superior_2013. pdf. Acesso em: 29/04/2015.

LAPEYRE, Nathalie; Le FEUVRE, Nicky. "Féminisation du corps médical et dynamiques profisionelles dans le champs de lasanté". Revue Française des Affaires Sociales, Paris, Ministère des Affaires sociales, de la Santé et des Droits des femmes, v. I, n. 1, p. 59-81, 2005.

LIMA, Betina Stefanelo. "O labirinto de cristal: as trajetórias de cientistas na Física". Revista Estudos Feministas, Florianópolis, v. 21, n. 3, p. 883-903, 2013.

LOPES, Maria Margaret. "'Aventureiras' nas ciências: refletindo sobre gênero e história das ciências naturais no Brasil". Cadernos Pagu, Campinas, n. 10, p. 345-368, 1998.

"Sobre convenções em torno de argumentos de autoridade". Cadernos Pagu, Campinas, n. 27, p. 35-61, 2006a.

"'Vencer barreiras', até quando? Aspectos da trajetória científico-políica de Bertha Maria Júlia Lutz (1894-1976)". In: SANTOS, Lucy Woellner dos et al. (Orgs.). Ciência, Tecnologia e Gênero. Desvelando o feminino na construção do conhecimento. Londrina: IAPAR, 2006b. p. 203-232.

LOMBARDI, Maria Rosa. Perseverança e resistência: a engenharia como profissão feminina. 2005. 292f. Tese (Doutorado em Educação), Faculdade de Educação, Unicamp, São Paulo.

"A engenheira brasileira contemporânea e a contribuição das mulheres nas mudanças recentes no campo profissional". Revista Tecnologia e Sociedade, Curitiba, n. 2, 2006a. Disponível em: www.ppgte.ct.utfpr.edu.br/. Acesso em: 07/04/2015.

."Engenheira \& Gerente: desafios enfrentados por mulheres em posições de comando na área tecnológica". Revista Tecnologia e Sociedade, Curitiba, n. 3, 2006a. Disponível em: www.ppgte.ct.utfpr.edu.br/. Acesso em: 05/04/2015.

As mulheres nas Forças Armadas brasileiras: a Marinha do Brasil. São Paulo: Fundação Carlos Chagas, 2008. 
MACHADO, Maria Helena (Coord.). Os médicos no Brasil: um retrato da realidade. Rio de Janeiro: Fiocruz, 1997.

MACHADO, Maria do Céu Soares. "A feminização da medicina". Análise Social, Lisboa, v. 38, n. 166, p. 127-137, 2003.

MELO, Hildete Pereira de. "Um olhar de gênero sobre o sistema de concessão de Bolsas de Pesquisa no CNPq - 2001/2008". In: BRASIL. Presidência da República. Secretaria Especial de Políticas para as Mulheres. Pensando Gênero e Ciência. Encontro Nacional de Núcleos e Grupos de Pesquisa - 2009, 2010. Brasília, SEPM, 2010. p. 176-191.

; CASEMIRO, Maria Carolina Pereira. "A Ciência no Feminino: uma análise da Academia Nacional de Medicina e da Academia Brasileira de Ciência". Revista Rio de Janeiro, Rio de Janeiro, UERJ/Fiocruz, p. 117-134, 2004.

; LASTRES, Helena Maria Martins. "Ciência e Tecnologia numa perspectiva de Gênero: o caso do CNPq". In: SANTOS, Lucy Woelner dos et al. Ciência, Tecnologia e Gênero. Desvelando o feminino na construção do conhecimento. Londrina: IAPAR, 2006. p.129160.

; OLIVEIRA, André Barbosa. "A produção científica brasileira no feminino". Cadernos Pagu, Campinas, n. 27, p. 301-331, 2006.

; RODRIGUES, Lígia Maria. Pioneiras das Ciências no Brasil. Rio de Janeiro: SBPC, 2006.

MILLAN, Luiz Alberto. Vocação Médica. Um estudo de Gênero. São Paulo: Casa do Psicólogo, 2005.

MINELLA, Luzinete Simões. "Pioneiras no campo da Medicina: trajetórias nas interseções". In: SEMINÁRIO INTERNACIONAL FAZENDO GÊNERO 10 -DESAFIOS ATUAIS DOS FEMINISMOS. 16 a 20 de setembro de 2013, Florianópolis/SC. Anais... Florianópolis, Universidade Federal de Santa Catarina, 2013a. Disponível em www.fazendogenero.ufsc.br/10/site/ anaiscomplementares. Acesso em: 25/05/2016.

"Temáticas prioritárias no campo de Gênero e Ciências no Brasil: raça/etnia, uma lacuna?". Cadernos Pagu, Campinas, n. 40, p. 95-140, 2013b.

. "Graduação em Medicina no Nordeste e no Sul do Brasil: um enfoque interseccional sobre o perfil dos/as estudantes". In: ; SARDENBERG, Cecília (Orgs.). Gênero e Ciências: mulheres em novos campos. Salvador: EDUFBA, 2015. p. 126-144. [no prelo]

MOTT, Maria Lúcia de Barros. "Madame Durocher: modista e parteira". Revista Estudos Feministas, Rio de Janeiro, v. 2, n. 3, p. 101-116, 1994.

Parto, parteiras, parturientes: Mme. Durocher e sua época. São Paulo: Faculdade de Filosofia, Letras e Ciências Humanas, Universidade de São Paulo, 1998. "Gênero, medicina e filantropia: Maria Renotte e as mulheres na construção da nação". Cadernos Pagu, Campinas, n. 24, p. 41-67, 2005.

; MUNIZ, M. A.; ALVES, O. S. F.; MAESTRINI, K.; SANTOS, T. "Médicos e médicas em São Paulo e os Livros de Registros do Serviço de Fiscalização do Exercício Profissional (18921932)". Ciência \& Saúde Coletiva, Rio de Janeiro, v. 13, p. 853-868, 2008.

OLINTO, Gilda. "A inclusão das mulheres nas carreiras de ciência e tecnologia no Brasil". Inclusão Social, Brasília, v. 5, n. 1, p. 68-77, 2011.

OECD - ORGANISATION FOR ECONOMIC CO-OPERATION AND DEVELOPMENT. OECD health data 2009: comparing health statistics across OECD countries, 2009. Disponível em: http://www.oecd.org/document/57/0,3746,en_21571361_44315115_43220022_1_1_1_ 1,00.html. Acesso em: 02/04/2015.

PISCITELLI, Adriana. "Interseccionalidade, categorias de articulação e experiências de migrantes brasileiras". Sociedade e Cultura, Goiânia, v. 11, n. 2, p. 263-274, 2008. 
RAGO, Elisabeth Juliska. Outras falas: feminismo e medicina na Bahia (1836-1931). São Paulo: Annablume/FAPESP, 2007.

RISKA, Elianne. Medical Careers and Feminist Agendas. American, Scandinavian and Russian Women Physician. New York: Aldine de Gruyter, 2001.

ROSENDE, Magdalena. Parcours féminins et masculins spécialisation en medicine. Genève: Seismo, 2008.

SANTOS, Tania Steren dos. "Gênero e carreira profissional na Medicina". Mulher e Trabalho, Porto Alegre, v. 4, p. 73-88, 2004.

SCHEFFER, Mário; CASSENOTE, Alex Jones F. "A feminização da medicina no Brasil”. Revista Bioética, Brasília, v. 21, n. 2, p. 268-277, 2013.

SCHIEBINGER, Londa. O Feminismo mudou a ciência?. Bauru: EDUSC, 2001.

SCHPUN, Monica R. "Entre feminino e masculino: a identidade política de Carlota Pereira de Queiróz". Cadernos Pagu, Campinas, n. 12, p. 331-377, 1999.

"A médica Carlota Pereira de Queiróz e seus colegas: amizade e relações profissionais em meio predominantemente masculino". In: MONTEIRO, Yara Nogueira (Org.). História da saúde: olhares e veredas. São Paulo: Instituto de Saúde, 2010. p. 239-250.

STOLCKE, Verena. "O enigma das interseções: classe, 'raça', sexo e sexualidade: a formação dos impérios transatlânticos do século XVI ao século XIX". Revista Estudos Feministas, v. 14, n. 1, p. 15-42, 2006.

TABAK, Fanny. O Laboratório de Pandora. Estudos sobre a ciência no feminino. Rio de Janeiro: Garamond, 2002.

TRIGO, Maria Helena Bueno. "A mulher universitária: códigos de sociabilidade e relações de gênero". In: BRUSCHINI, Cristina; SORJ, Bila (Orgs.). Novos Olhares: mulheres e relações de gênero no Brasil. São Paulo: Marco Zero, 1994. p. 89-110.

TAVARES-NETO, José. Formados de 1812 a 2008 pela Faculdade de Medicina da Bahia. Academia de Medicina de Feira de Santana: Feira de Santana, 2008.

YANNOULAS, Silvia Cristina (Org.). Trabalhadoras: análise da feminização das profissões e ocupações. Brasília: Abaré, 2013.

UNIVERSIDADE FEDERAL DA BAHIA. Pró-Reitoria de Planejamento e Administração. Perfil sócioeconômico dos candidatos inscritos e classificados no concurso vestibular da UFBA. 2005-2008. Salvador: UFBA, 2008. Disponível em: www.ufba.br/. Acesso em: 13/04/2015.

UNIVERSIDADE FEDERAL DE SANTA CATARINA. Relatório de Avaliação do Programa de Ações Afirmativas. Florianópolis: UFSC, 2012.

VANIN, lole Macedo. As damas de branco na biomedicina baiana (1879-1949): médicas, farmacêuticas e odontólogas. 2008. Tese (Doutorado em História), Universidade Federal da Bahia, Salvador.

[Recebido em 31/05/2016

e aprovado em 26/09/2016]

Medicine and Feminization in Brazilian Universities: Gender and Intersections

Abstract: This article analyzes the profile socio-economic, generational and ethnic of the students and aspirants students to Medicine graduation courses in two Brazilian states: Bahia and Santa Catarina, between 2005 and 2015. The intersectional approach draws upon the authors of contributions Gender field and Sciences, as well as studies on the participation of women in the medical area in Brazil, in order to further contribute to the debate about the feminisation of the area. Considering regional interferences in the setting of the profiles found in public universities (UFBA and UFSC), as well as the private network universities (FTC and EBMSP in Bahia and UNIVALI and UNESC in Santa Catarina), the research methodology included the collection of secondary data 
(about sex, household income, age, color and origin), document analysis and review of the literature. Keywords: Gender; Intersections; Feminization; Medicine

Luzinete Simões Minella (simoesluzinete@gmail.com) é graduada e mestre em Ciências Sociais pela UFBA, doutora em Sociologia pela Universidad Nacional Autónoma de México (UNAM,1989). Realizou estágio de pós-doutorado no Núcleo de Estudos de População da Universidade Estadual de Campinas (NEPO/Unicamp). Atualmente é professora adjunta IV aposentada da Universidade Federal de Santa Catarina, atuando no PPG Interdisciplinar em Ciências Humanas e no PPG em Sociologia Política. Pesquisadora do Instituto de Estudos de Gênero (IEG) da mesma universidade.

1128 Estudos Feministas, Florianópolis, 25(3): 1111 -1128, setembro-dezembro/2017 